\title{
Establishing a surrogate model for inactivation of enveloped viruses to screen viral clearance conditions during biotherapeutics process development
}

\author{
Hasin Feroz ${ }^{1}$, Daniel Cetnar ${ }^{2}$, Robert Hewlett ${ }^{1}$, Satish Sharma ${ }^{1}$, Melissa Holstein ${ }^{1}$, \\ Sanchayita Ghose ${ }^{3}$, and Zheng Jian $\mathrm{Li}^{3}$ \\ ${ }^{1}$ Bristol-Myers Squibb Co \\ ${ }^{2}$ Penn State University Park \\ ${ }^{3}$ Bristol-Myers Squibb
}

April 6, 2021

\begin{abstract}
Viral surrogates to screen for virus inactivation (VI) can be a faster, cheaper and safer alternative to third-party testing of pathogenic BSL2 (Biosafety Level 2) model viruses. Although the bacteriophage surrogate, Ø6, has been used to assess low pH BSL2 VI, it has not been used for evaluation of detergent-mediated VI. Furthermore, Ø6 is typically assayed through host cell infectivity which introduces the risk of cross-contaminating other cell lines in the facility. To circumvent contamination, we developed an in-house RT-qPCR (reverse transcriptase quantitative polymerase chain reaction) assay for selective detection of active $\varnothing 6$ from a population of live and dead phage. The RT-qPCR assay was used to evaluate $\varnothing 6$ inactivation in cell culture fluid of monoclonal antibody and fusion protein. Complementary $\varnothing 6$ infectivity was also conducted at a third-party testing facility. The $\varnothing 6$ RT-qPCR and infectivity data was modeled against VI of three BSL2 viruses, X- MuLV, A- MuLV and HSV-1 in corresponding therapeutics. Both Ø6 methods demonstrate that any VI agent showing Ø6 clearance of [?] 2.5 logs would demonstrate complete BSL2 VI of [?] 4.0 logs. Compared to BSL2 virus testing, this in-house O6 RT-qPCR tool can screen VI agents at $5 \%$ the cost and a turnaround time of 2-3 days versus 4-7 months.
\end{abstract}

\section{Hosted file}

VI Phage Manuscript.pdf available at https://authorea.com/users/406120/articles/516912establishing-a-surrogate-model-for-inactivation-of-enveloped-viruses-to-screen-viralclearance-conditions-during-biotherapeutics-process-development 


\section{A. Phage inactivation}

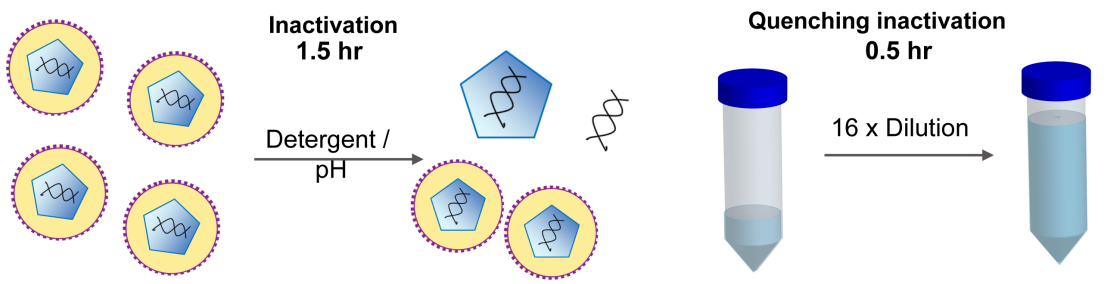

\section{B. Phage RT-qPCR assay}
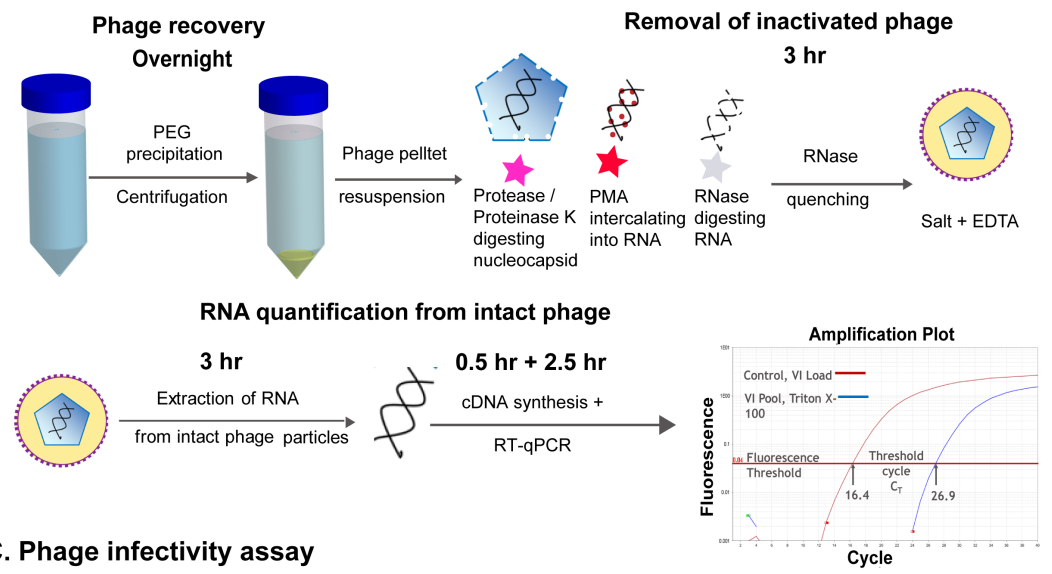

Serial dilution of phage-spiked sample Plating phage with host cell Counting plaque forming units $2 \mathrm{hr}$

$3 \mathrm{hr}$

5 days

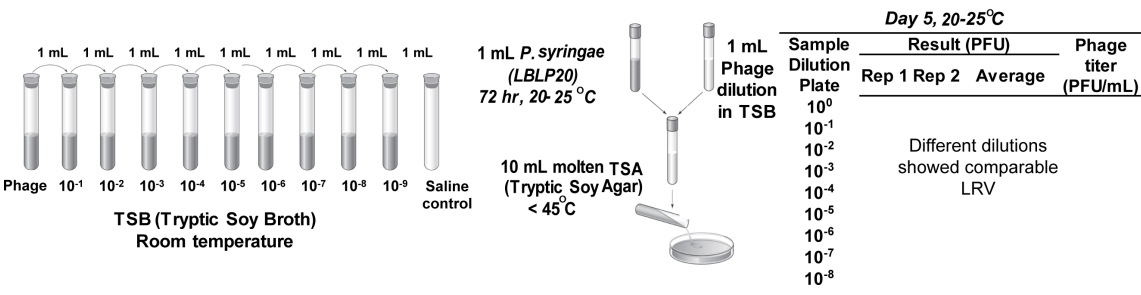

A

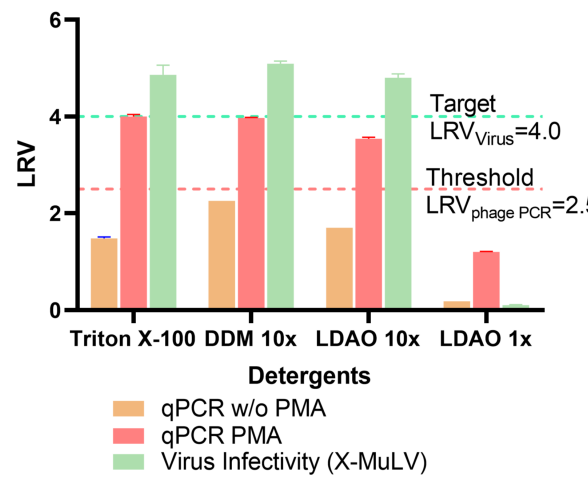

B

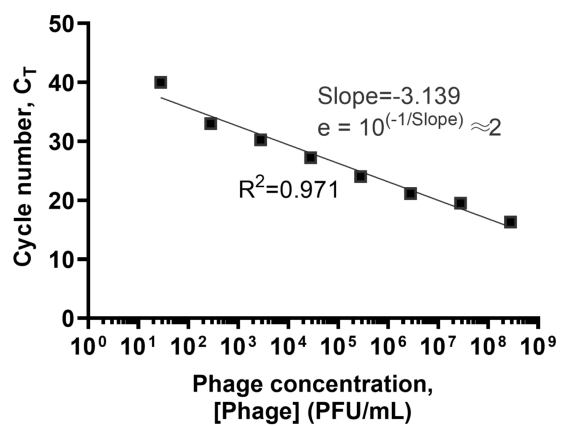




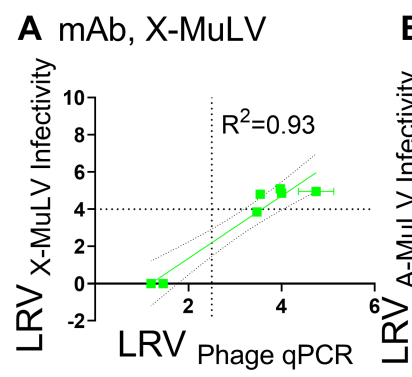

D

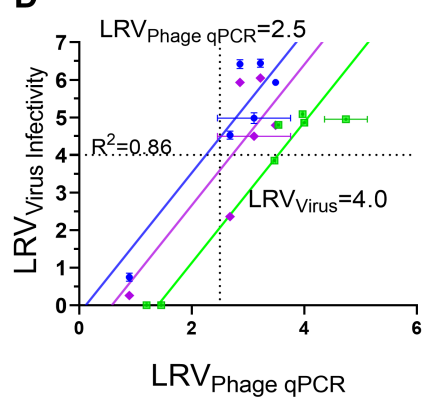

mAb, X-MuLV

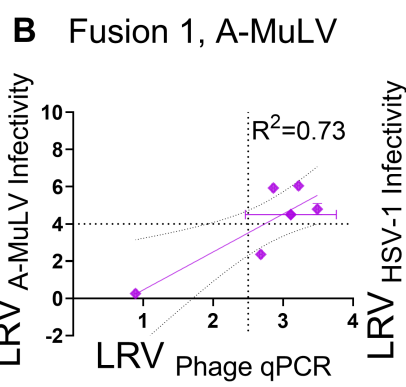

E

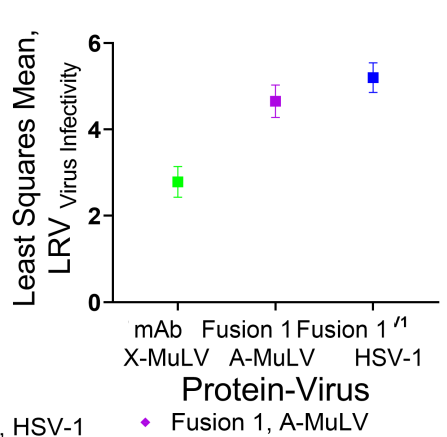

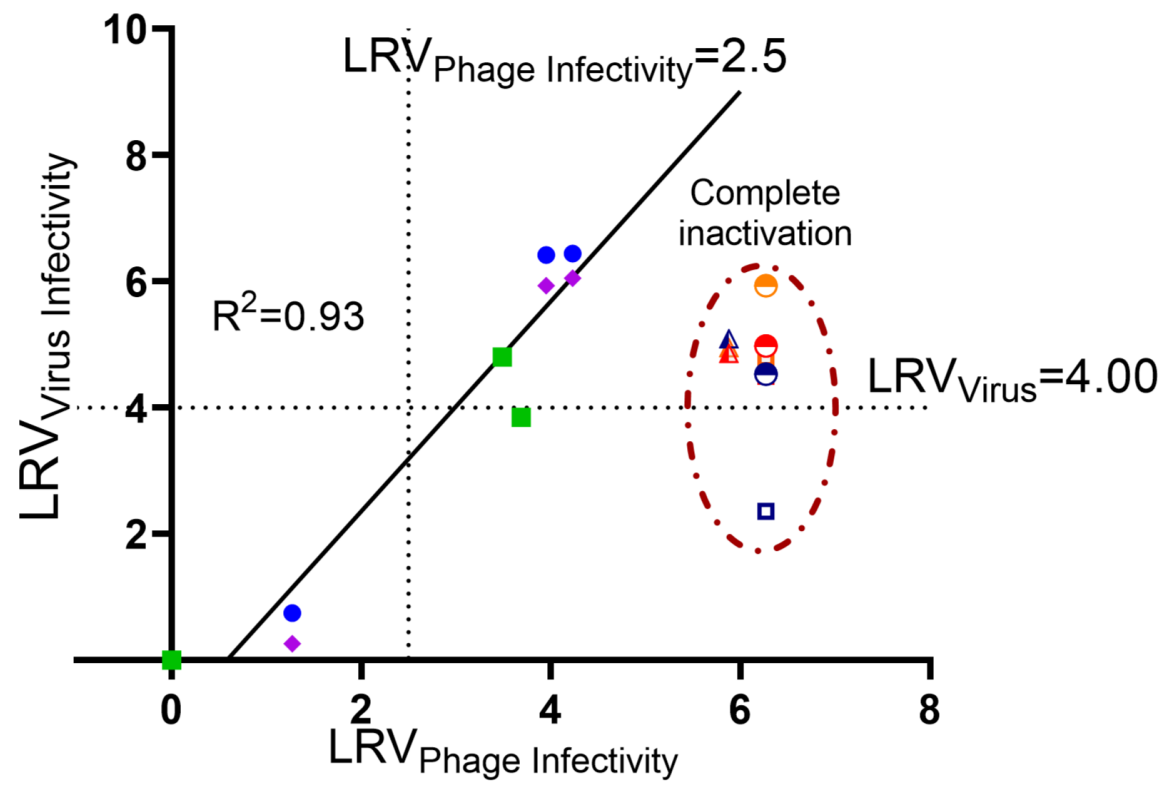

Complete clearance

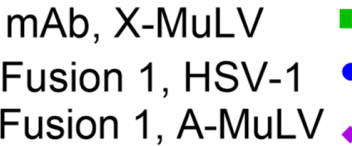

Triton X-100 Ecosurf DDM

$\begin{array}{ccc}\Delta & \Delta & \Delta \\ \ominus & \ominus & \theta \\ \square & \square & \square\end{array}$




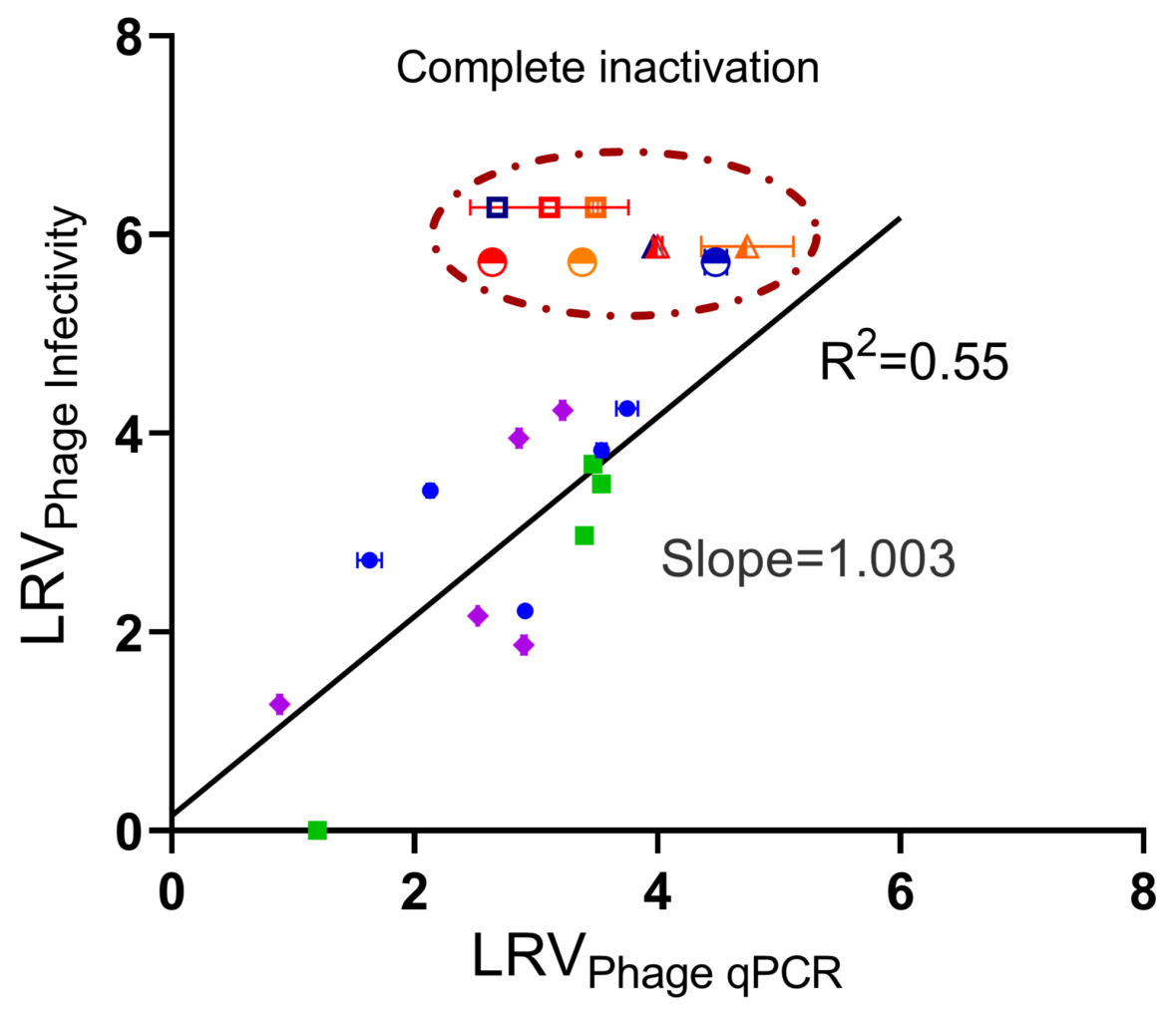

Complete clearance Triton X-100 Ecosurf DDM

$\begin{array}{lllll}\text { mAb } & \Delta & \Delta & \Delta \\ \text { Fusion 2 } & \bullet & \ominus & \ominus & \ominus \\ \text { Fusion 1 } & \bullet & \square & \text { व }\end{array}$

